OPEN ACCESS

Edited by:

Xiao-Jun Ji,

Nanjing Tech University, China

Reviewed by:

Farshad Darvishi,

Alzahra University, Iran

Zongjie Dai,

Tianjin Institute of Industrial Biotechnology (CAS), China

$$
\begin{array}{r}
\text { *Correspondence: } \\
\text { Yu-Sin Jang } \\
\text { jangys@gnu.ac.kr } \\
\text { Sang Yup Lee } \\
\text { leesy@kaist.ac.kr }
\end{array}
$$

Specialty section:

This article was submitted to Industrial Biotechnology,

a section of the journal

Frontiers in Bioengineering and

Biotechnology

Received: 06 August 2021 Accepted: 11 October 2021

Published: 25 October 2021

Citation:

Jang $Y$-S, Seong HJ, Kwon SW, Lee Y-S, Im JA, Lee HL, Yoon YR and

Lee SY (2021) Clostridium

acetobutylicum atpG-Knockdown

Mutants Increase Extracellular $\mathrm{pH}$ in

Batch Cultures.

Front. Bioeng. Biotechnol. 9:754250.

doi: 10.3389/fbioe.2021.754250

\section{Clostridium acetobutylicum atpG-Knockdown Mutants Increase Extracellular pH in Batch Cultures}

\author{
Yu-Sin Jang ${ }^{1 *}$, Hyeon Jeong Seong ${ }^{1}$, Seong Woo Kwon ${ }^{1}$, Yong-Suk Lee ${ }^{1}$, Jung Ae $\mathrm{Im}^{2}$, \\ Haeng Lim Lee ${ }^{1}$, Ye Rin Yoon ${ }^{1}$ and Sang Yup Lee ${ }^{2 *}$ \\ ${ }^{1}$ Division of Applied Life Science (BK21), Department of Applied Life Chemistry, Institute of Agriculture and Life Science (IALS), \\ Gyeongsang National University, Jinju, South Korea, ${ }^{2}$ Department of Chemical and Biomolecular Engineering (BK21 Plus \\ Program), BioProcess Engineering Research Center, Institute for the BioCentury, Korea Advanced Institute of Science and \\ Technology (KAIST), Daejeon, South Korea
}

ATPase, a key enzyme involved in energy metabolism, has not yet been well studied in Clostridium acetobutylicum. Here, we knocked down the atpG gene encoding the ATPase gamma subunit in C. acetobutylicum ATCC 824 using a mobile group II intron system and analyzed the physiological characteristics of the atpG gene knockdown mutant, 8242866KD. Properties investigated included cell growth, glucose consumption, production of major metabolites, and extracellular $\mathrm{pH}$. Interestingly, in 2-L batch fermentations, 8242866KD showed no significant difference in metabolite biosynthesis or cell growth compared with the parent ATCC 824. However, the $\mathrm{pH}$ value in 824-2866KD cultures at the late stage of the solventogenic phase was abnormally high (pH 6.12), compared with that obtained routinely in the culture of ATCC 824 (pH 5.74). This phenomenon was also observed in batch cultures of another C. acetobutylicum, BEKW-2866KD, an atpGknockdown and pta-buk double-knockout mutant. The findings reported in this study suggested that ATPase is relatively minor than acid-forming pathway in ATP metabolism in C. acetobutylicum.

Keywords: Clostridium acetobutylicum, ATPase, atpG, knockdown, extracellular pH

\section{INTRODUCTION}

Clostridium acetobutylicum is a strictly anaerobic, gram-positive bacterium that survives in hostile environments by producing endospores (Shao et al., 2007). C. acetobutylicum possesses industrially applicable metabolic properties, notably including the production of organic solvents, such as acetone, butanol, and ethanol (Kwon et al., 2020; Shin et al., 2021). C. acetobutylicum produces the solvents through biphasic pathway, which is divided into an acidogenic phase and a solventogenic phase (Shao et al., 2007; Im et al., 2021). During the acidogenic phase, which corresponds to the initial growth phase, most carbon sources are used to produce acetate, butyrate, and carbon dioxide (Jang et al., 2012). As cell growth enters the stationary phase, the metabolism of C. acetobutylicum shifts to the solventogenic phase (Lütke-Eversloh, 2014), during which organic acids are reassimilated, and most of the carbon sources are used to produce butanol, acetone, and ethanol as final products (Jang et al., 2012).

The reason for this biphasic fermentation is closely related to the energy and redox metabolism in C. acetobutylicum (Figure 1) (Jang et al., 2014a; Lütke-Eversloh, 2014). In these bacteria, ATP is primarily produced from glucose through glycolysis (Externbrink et al., 2000). During the initial 


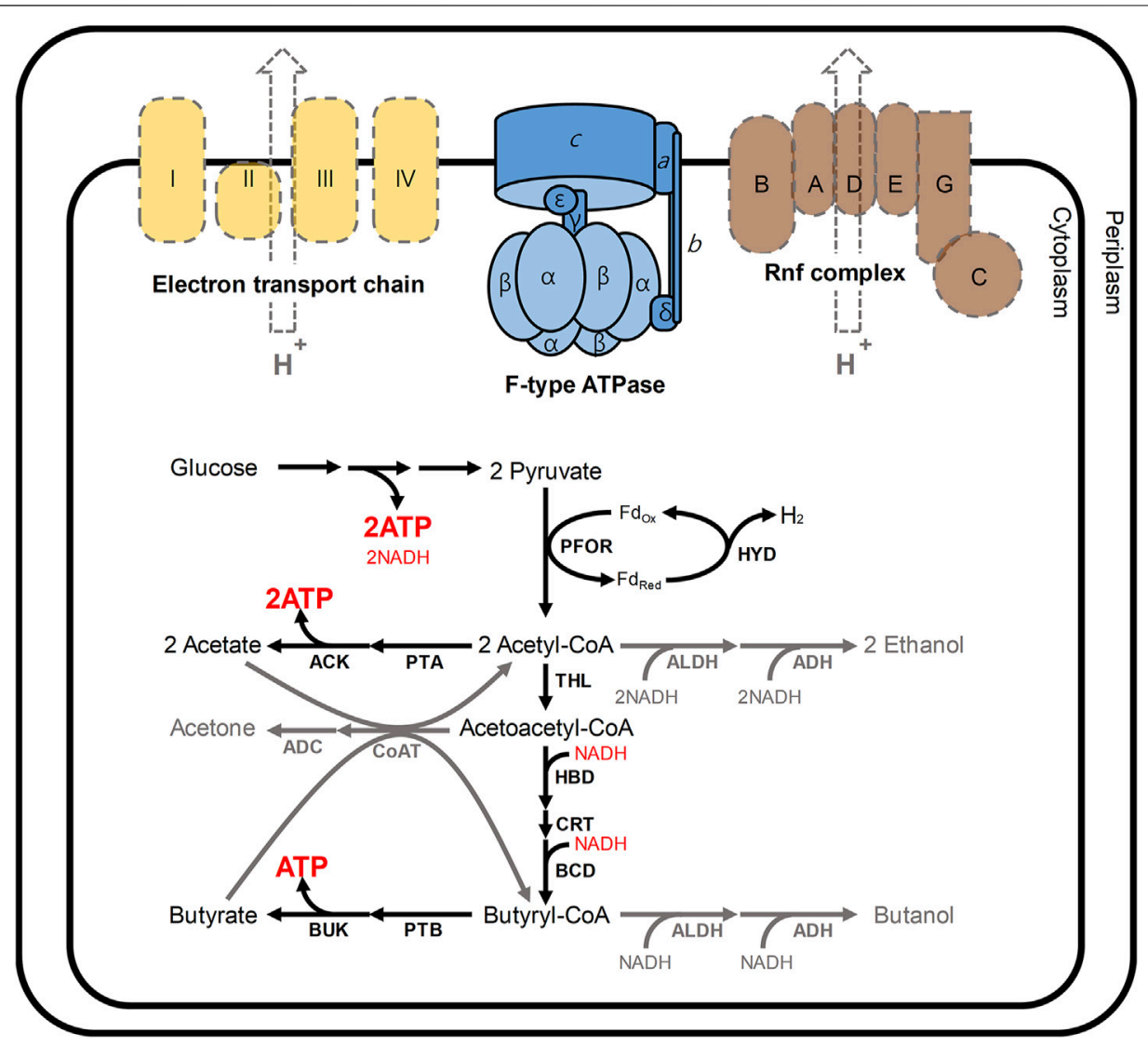

FIGURE 1 | Schematic presentation of the energy and redox metabolism in C. acetobutylicum. In C. acetobutylicum, F-type ATPase is encoded by the atp operon (atpIBEFHAGDC). In general, F-type ATPases require the proton motive force to produce ATP; however, neither the electron transport chain (yellow) nor Rnf complex (brown) has been reported in C. acetobutylicum (Tremblay et al., 2012). The Rnf complex is commonly reported in other clostridia such as Clostridium beijerinkii, Clostridium saccharobutylicum and Clostridium saccharoperbutylacetonicum, but it is usual that the Rnf is not found in C. acetobutylicum (Poehlein et al., 2017). In this situation, meanwhile, 2 mol of ATP are produced from a glucose through glycolysis. During the initial growth phase (namely, acidogenic phase), additional ATP is produced through the routes for the production of acetate and butyrate. At that time, NADH could be re-oxidized via two routes: 1) a cascade reaction for butyrate formation involving 3-hydroxybutyryl-CoA dehydrogenase (HBD) and butyryl-CoA dehydrogenase (BCD); 2) hydrogen production reaction catalyzed by hydrogenase ( $H Y D$ ) coupled with ferredoxin oxidoreductase (PFOR). During solventogenic phase (gray arrows), the function of hydrogenase is turned-off, and $\mathrm{NAD}^{+}$is regenerated by 4 (HBD, BCD, ALDH, and ADH) and 2 (ALDH and ADH) dehydrogenases for butanol and ethanol biosynthesis, respectively. Abbreviations: ACK, acetate kinase; ADC, acetoacetate decarboxylase; ADH, alcohol dehydrogenase; ALDH, aldehyde dehydrogenase; BUK, butyrate kinase, CoAT, CoA transferase; CRT, crotonase; THL, thiolase; PTA, phosphotransacetylase; and PTB, phosphotransbutyrylase.

growth phase in C. acetobutylicum, additional ATP is produced through substrate-level phosphorylation, which is coupled to the production of acetate and butyrate (Externbrink et al., 2000). At that time, to regenerate $\mathrm{NAD}^{+}, \mathrm{NADH}$ could be oxidized via not only two enzymes 3-hydroxybutyryl-CoA dehydrogenase (HBD) and butyryl-CoA dehydrogenase (BCD) responsible for butyrate formation, but also hydrogenase (HYD) coupled with ferredoxin oxidoreductase (PFOR; Figure 1) (Du et al., 2021; Jiang et al., 2021). As the acidogenic phase progresses, the external $\mathrm{pH}$ is continuously lowered to nearby 4.5 , and $\mathrm{NADH}$ also accumulates, both of which have adverse effects on $C$. acetobutylicum (Jang et al., 2012; Lütke-Eversloh, 2014). At this point, the metabolism of C. acetobutylicum shifts from the acidogenic phase to the solventogenic phase (Jang et al., 2012; Li et al., 2020; Thi et al., 2020). After such phase transition, the function of hydrogenase is turned-off, and $\mathrm{NAD}^{+}$is regenerated by 4 and 2 dehydrogenases for butanol and ethanol biosynthesis, respectively (Tremblay et al., 2012; Fast and Papoutsakis, 2018) (see Figure 1 for details). Continuous acid re-assimilation and carbon flux toward solvent production cause the lowered external pH to rise (Kim et al., 2020; Li et al., 2020).

Despite such perfect metabolism for energy and redox regulation through biphasic fermentation, the atp operon encoding ATPase was reported in C. acetobutylicum genome (Nölling et al., 2001; Cho et al., 2017). The fully sequenced atp operon in $C$. acetobutylicum has been shown to include the atpIBEFHAGDC (F-type ATPase) (Externbrink et al., 2000). F-type ATPases, which are conjugated to the inner membrane in microbes, generally mediate ATP synthesis through oxidative phosphorylation (Externbrink et al., 2000; Mukherjee and Warshel, 2015; Zharova and Vinogradov, 2017; Kang et al., 2019). F-type ATPases require the proton motive force (PMF) to produce ATP from ADP and inorganic phosphate (Pi); however, neither the electron transport chain nor Rnf complex 
has been reported in C. acetobutylicum (Tremblay et al., 2012; Shin et al., 2021) (Figure 1). Although physiological effects of disrupting ATPase have been analyzed in other organisms, such as Escherichia coli (Jensen and Michelsen, 1992; Causey et al., 2003; Shah and Duncan, 2015; Burger et al., 2020), Lactococcus lactis (Koebmann et al., 2000; Koebmann et al., 2002a), Rhodobacter capsulatus (Borghese et al., 1998), Saccharomyces cerevisiae (Weber et al., 1995; Zhang and Zhang, 2019), Corynebacterium glutamicum (Sekine et al., 2001; KochKoerfges et al., 2012), and Bacillus subtilis (Santana et al., 1994), the consequences of ATPase mutation in $C$. acetobutylicum ATCC 824 have not yet been investigated. Here, to reveal the main function of F-type ATPase in $C$. acetobutylicum, we constructed ATPase-knockdown strains and performed a physiological characterization of resulting ATPase-knockdown strains.

\section{MATERIALS AND METHODS}

\section{Bacterial Strains, Plasmids, and Culture Conditions}

E. coli strains and recombinants were grown in Luria-Bertani (LB) broth at $37^{\circ} \mathrm{C}$ (An et al., 2020; Lone et al., 2020). C. acetobutylicum ATCC 824 and the engineered strain BEKW and mutants were grown in clostridial growth medium (CGM) or 2X YTG agar in an anaerobic chamber (Forma Scientific, Marietta, $\mathrm{OH}$, United States) under 4\% hydrogen and 96\% nitrogen at $37^{\circ} \mathrm{C}$ (Jang et al., 2012). Ampicillin $(50 \mu \mathrm{g} / \mathrm{ml}$ ), chloramphenicol $(34 \mu \mathrm{g} / \mathrm{ml})$, or erythromycin $(40 \mu \mathrm{g} / \mathrm{ml})$ was added to the medium, as required.

\section{Construction of Knockdown Mutants}

The mobile group II intron system was used to construct atpGknockdown mutants of C. acetobutylicum (Heap et al., 2007; Shao et al., 2007; Jang et al., 2012; Jang et al., 2014b; Kim et al., 2015; Kwon et al., 2020). The atpG-targeted intron for knockdown was amplified by overlap extension PCR using the following primers: 2866-IBS, 5' -AAAAAAGCTTATAATTATCCTTAATAGCCGA CCGTGTGCGCCCAGATAGGGTG-3'; 2866-EBS1， 5'-CAG ATTGTACAAATGTGGTGATAACAGATAAGTCGACCGTG CTAACTTACCTTTCTTTGT-3'; 2866-EBS2, 5' CAAGTTTCTAATTTCGGTTGCTATCCGATAGAGGAAAGT GTCT-3'; EBS universal, 5'-CGAAATTAGAAACTTGCGTTC AGTAAAC-3' (Supplementary Table S1). The amplified PCR fragment $(\sim 0.5 \mathrm{~kb})$ was double-digested using restriction enzymes BsrGI and HindIII, and then ligated into pCACYS3 (Jang et al., 2012) digested using the same enzymes, yielding the recombinant plasmid, pCAC2866KD. Plasmid pCAC2866KD was consecutively transformed into E. coli TOP10 (pAN1) containing the plasmid pAN1, which harbors the methyltransferase gene, $\varphi 3$ TI (Mermelstein and Papoutsakis, 1993). Thus, the recombinant plasmid, pCAC2866KD, is methylated by the methyltransferase in the resulting $E$. coli strain. C. acetobutylicum ATCC 824 and its pta-buk double mutant BEKW (Jang et al., 2012) were subsequently transformed with the methylated recombinant plasmid, yielding the atpG-knockdown mutant strains, $824-2866 \mathrm{KD}$ and BEKW-2866KD, respectively. The resulting atpG-knockdown mutants, in which the targeted intron was inserted in the sense strand, were validated by PCR using primers atpG-F and atpG-R (Supplementary Table S1). The intron insertion into the target site on the atpG gene was further confirmed by sequencing the DNA fragments obtained from PCR with primers atpG-seq-F and atpG-seq-R using total DNA of the mutant (Supplementary Table S1).

\section{Batch Fermentation}

C. acetobutylicum ATCC 824 and its mutants were inoculated into 500-ml Erlenmeyer flasks containing $200 \mathrm{ml} \mathrm{CGM}$ and then cultured anaerobically to an optical density at $600 \mathrm{~nm}\left(\mathrm{OD}_{600}\right)$ of 1.0 at $37^{\circ} \mathrm{C}$ (Jang et al., 2012). The resulting seed cultures were transferred into a 5-L Liflus GX bioreactor (Biotron, Gyunggi-do, South Korea) containing $1.8 \mathrm{~L}$ CGM for fermentation. The bioreactor was set at an agitation speed of $200 \mathrm{rpm}$, a nitrogen gas flow rate of $0.25 \mathrm{vvm}$, and a temperature of $37^{\circ} \mathrm{C}$. The $\mathrm{pH}$ was automatically maintained above 5.0 with ammonia solution but was not controlled when $\mathrm{pH}$ became higher than the set value. Samples were periodically withdrawn from the culture medium for analysis of cell growth and concentrations of glucose, organic acids, and organic solvents.

\section{Analytical Methods}

Samples were collected for monitoring cell growth, glucose consumption, $\mathrm{pH}$, and production of metabolites, including acetate, butyrate, acetone, ethanol, and butanol. Batch fermentations of each strain were independently performed in duplicate. Cell growth was monitored by measuring $\mathrm{OD}_{600}$ using an Ultrospec 3000 spectrophotometer (Pharmacia Biotech, Uppsala, Sweden). The concentrations of acetate, butyrate, and glucose were determined using a high-performance liquid chromatography (HPLC) system (Prostar; Varian, Palo Alto, CA, United States) equipped with a packed column (Metacarb 87H; MetaChem Technologies, Torrance, CA, United States) and refractive index detector (RI-27; Shodex, Japan). The mobile phase consisted of $0.01 \mathrm{~N} \mathrm{H}_{2} \mathrm{SO}_{4}$ (Im et al., 2019; Chun and Sang, 2020; Lee et al., 2020). The concentrations of acetone, butanol, and ethanol were determined using a gas chromatography system (Agilent 7890; Agilent Technologies, California, United States) equipped with a packed column (80/ 120 Carbopack BAW glass column; Supelco, Bellefonte, PA, United States) and flame ionization detector (Jang et al., 2012; Baek et al., 2019). Helium gas was used for the mobile phase.

\section{RESULTS AND DISCUSSION}

\section{Construction of the atpG-Knockdown C. acetobutylicum Mutants}

CAC2866 (atpG encoding ATPase gamma subunit), one of nine ATPase-coding genes found in C. acetobutylicum, is an important part of the ATPase enzyme (Externbrink et al., 2000). The ATPase gamma subunit forms the central shaft, which forms the connection between the $\mathrm{F}_{0}$ rotary motor and the $\mathrm{F}_{1}$ catalytic 

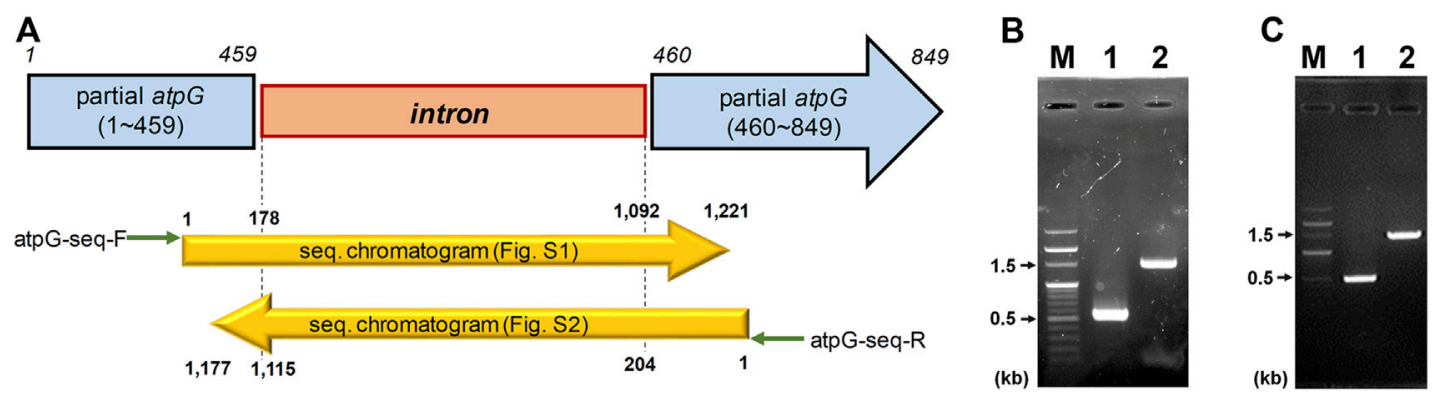

FIGURE 2 | Inactivation of C. acetobutylicum atpG gene by the intron insertion using mobile group II intron system. (A) Schematic diagram of the mutated atpG gene (blue) constructed by intron (orange) insertion. The intron was inserted between 459th and 460th nucleotides in the wild-type atpG gene, which was confirmed by sequencing using primers atpG-seq-F (green arrow) and atpG-seq-R (reverse green arrow; Supplementary Table S1). The mutated atpG gene was schematically aligned with DNA sequencing chromatograms (yellow arrows; see Supplementary Figures S1, S2 for detailed chromatogram). (B,C) Validation of the atpG gene mutation in strains 824-2866KD (B) and BEKW-2866KD (C). The atpG-knockdown mutants, 824-2866KD and BEKW-2866KD were validated by PCR using primers atpG-F and atpG-R (Supplementary Table S1). (B) M, 100-bp marker; lane \#1, ATCC 824; lane \#2, 824-2866KD. (C) M, 100-bp marker; lane \#1, BEKW; lane \#2, BEKW-2866KD.
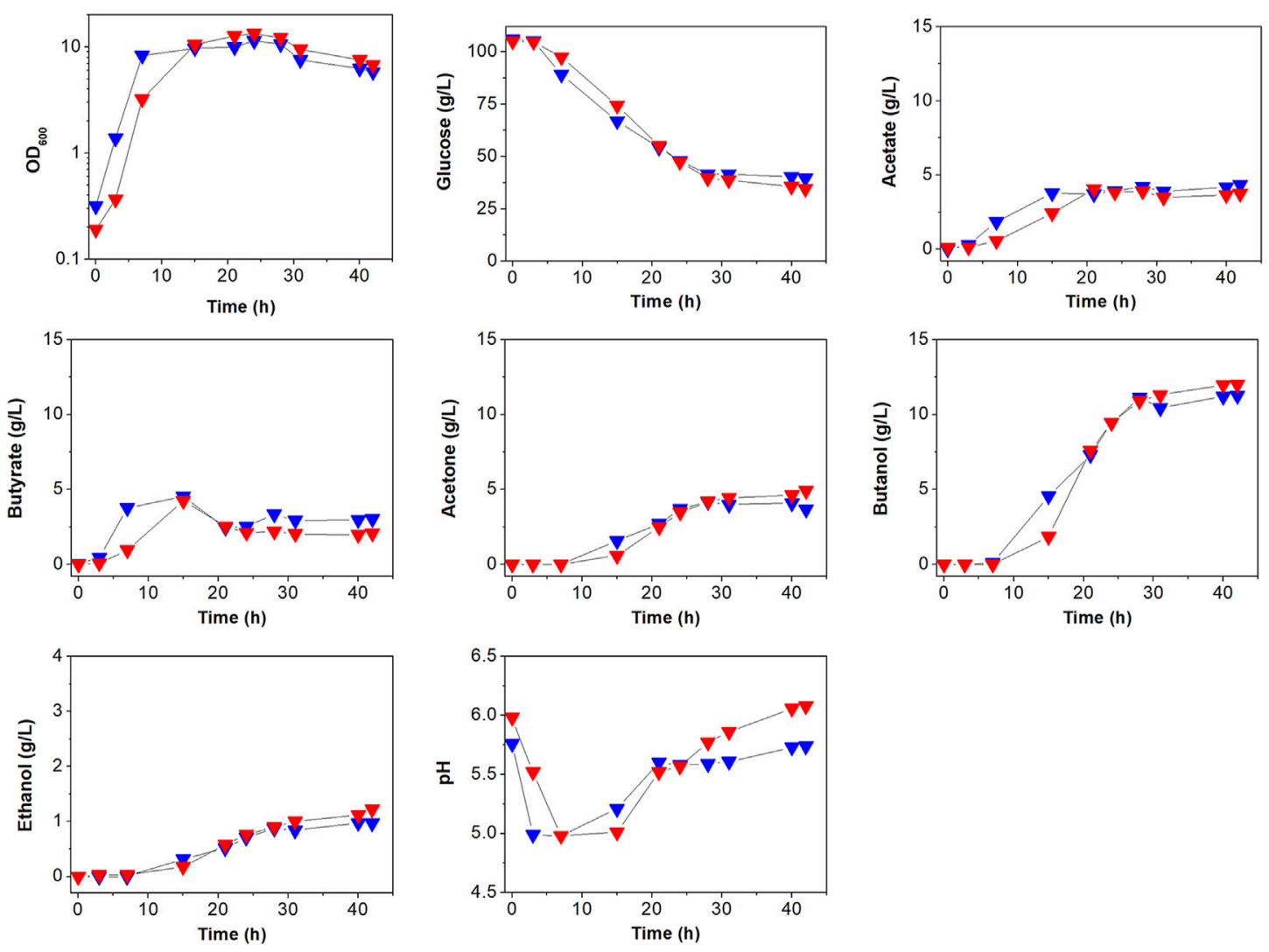

FIGURE 3 | Comparison of batch fermentation profiles between C. acetobutylicum ATCC 824 (blue) and its mutant 824-2866KD (red). Fermentation parameters are cell growth $\left(\mathrm{OD}_{600}\right)$ glucose consumption, acids (acetate and butyrate) production, and solvents (acetone, butanol, and ethanol) production. Merged version of batch fermentation profiles of $C$. acetobutylicum 824-2866KD are shown in Supplementary Figure S3A. Other reproduced bioreactor cultivation profiles are shown in Supplementary Figure S3B.

complex (Figure 1; Mukherjee and Warshel, 2015). Disruption of the gamma subunit of F-ATPase in other organisms decreases ATPase activity and ATP levels, resulting in cell growth inhibition and a shift in metabolism (Iwamoto et al., 1990; Shin et al., 1992; Lai-Zhang et al., 1999). Accordingly, to investigate the effects of ATPase knockdown on physiological 
characteristics of C. acetobutylicum, we constructed atpGknockdown mutant strains, 824-2866KD and BEKW-2866KD from wild-type ATCC 824 and the pta-buk double mutant BEKW strains, respectively, by using mobile group II intron system (Figure 2; Supplementary Figures S1, S2).

\section{Effects of atpG Knockdown on Cell Growth, Glucose Consumption, and Metabolite Production}

To see the effects of atpG knockdown on physiological characteristics, we first analyzed and compared cell growth between ATCC 824 and 824-2866KD (Figure 3; Supplementary Figure S3). There was no apparent difference in growth between ATCC 824 and 824-2866KD (Figure 3), even though disruption of ATPase is known to reduce ATPase activity and ATP level, which in turn inhibits cell growth in non-clostridia strains (Iwamoto et al., 1990; Ferrandiz and De La Campa, 2002; Causey et al., 2003; Cipriano et al., 2006; Kim et al., 2020).

The effects of atpG knockdown in C. acetobutylicum were also assessed by examining glucose consumption, which is known to be affected by ATP levels (Koebmann et al., 2002b; Dai et al., 2020). Glucose concentration decreased steadily during exponential and stationary phases in both ATCC 824 and 8242866KD (Figure 3; Supplementary Figure S3). After $28 \mathrm{~h}$, glucose consumption in ATCC 824 was $66.5 \mathrm{~g} / \mathrm{L}$ and was maintained at $39.5 \mathrm{~g} / \mathrm{L}$ (Figure 3). 824-2866KD showed a similar decrease in glucose consumption rate, which reached $70.5 \mathrm{~g} / \mathrm{L}$ at $28 \mathrm{~h}$ and was maintained at $34.5 \mathrm{~g} / \mathrm{L}$ (Figure 3). Thus, these results show no significant changes in glucose consumption in atpG-knockdown $C$. acetobutylicum mutant comparing with the parent ATCC 824 strain.

The effects of atpG knockdown were further investigated by analyzing the production of metabolites (Figure 3; Supplementary Figure S3). The highest concentrations of acetate and butyrate in $824-2866 \mathrm{KD}$ culture were $3.9 \mathrm{~g} / \mathrm{L}$ and $4.0 \mathrm{~g} / \mathrm{L}$, respectively, representing 91.6 and $88.9 \%$ of concentrations in ATCC 824 fermentation (Figure 3). During the solventogenic phase, the final concentrations of acetate and acetone in $824-2866 \mathrm{KD}$ were also similar to those in ATCC 824 (Figure 3). However, residual butyrate in the fermentation using 824-2866KD was slightly lower than that of the ATCC 824, with a difference of exactly $0.98 \mathrm{~g} / \mathrm{L}$ at the endpoint (Figure 3). The lack of change (or minor change) in acid and acetone concentrations indicates that acid re-assimilation is also not majorly affected by ATPase knockdown. The production of ethanol and butanol in 824-2866KD culture were $1.4 \mathrm{~g} / \mathrm{L}$ and $12.4 \mathrm{~g} / \mathrm{L}$, respectively, which were also similar to the corresponding concentrations of $1.0 \mathrm{~g} / \mathrm{L}$ and $11.3 \mathrm{~g} / \mathrm{L}$ in ATCC 824 fermentation (Figure 3). Previous studies have reported that disruption of ATPase shifts metabolic flux toward byproducts because ATPase-disrupted mutants produce ATP through substrate-level phosphorylation, not by oxidative phosphorylation (Koebmann et al., 2002a; Koebmann et al., 2002b). It seems that as most ATP in C. acetobutylicum is produced through substrate-level phosphorylation, the ATPase-knockdown mutant showed no significant changes in acidogenic or and solventogenic phases.

\section{Effect of atpG Knockdown on Extracellular pH}

The effect of atpG knockdown was also analyzed by comparing extracellular pH between ATCC 824 and 824-2866KD (Figure 3; Supplementary Figure S3). Throughout the entire fermentation period, the bioreactor controller adjusted the external $\mathrm{pH}$ to maintain it above 5.0. During the acidogenic phase, ATCC 824 and $824-2866 \mathrm{KD}$ reached $\mathrm{pH} 5.0$ and maintained it by adding ammonia solution to avoid decreasing $\mathrm{pH}$ values by the production of organic acids. During the subsequent solventogenic phase, $\mathrm{pH}$ rose as a result of acid re-assimilation in both ATCC 824 and $824-2866 \mathrm{KD}$ cultures (Figure 3). The $\mathrm{pH}$ rose steadily after $20 \mathrm{~h}$, reaching $\mathrm{pH} 5.74$ in ATCC 824 culture (Figure 3). On the other hand, $\mathrm{pH}$ rose steadily for more than $40 \mathrm{~h}$ in $824-2866 \mathrm{KD}$ culture, reaching a value of 6.12 at the late stage of the solventogenic phase, a value significantly higher than that in ATCC 824 fermentation (Figure 3). These results show that ATPase activity is affected to the extracellular $\mathrm{pH}$ in $C$. acetobutylicum fermentation.

\section{Effect of atpG Knockdown on Physiological Characteristics of $C$. acetobutylicum BEKW} Our previous work (Jang et al., 2012) showed that C. acetobutylicum BEKW exhibited higher butanol production $(16.0 \mathrm{~g} / \mathrm{L})$ than C. acetobutylicum ATCC 824 (11.8 g/L). Two enzymes including phosphotransacetylase and butyrate kinase encoded by the pta and $b u k$, respectively, operate primarily in the acidogenic phase to synthesize the organic acids, acetate and butyrate, respectively, in addition to producing ATP through substrate-level phosphorylation (Lütke-Eversloh, 2014). To determine the effects of atpG knockdown in BEKW, we cultured the mutant, BEKW-2866KD in 2-L bioreactor (Figure 4A; Supplementary Figure S4).

First, we compared cell growth and glucose consumption of BEKW-2866KD with that in BEKW. Glucose concentration decreased steadily during exponential and stationary phases in BEKW-2866KD (Figure 4A). Glucose consumption ceased by $38 \mathrm{~h}$ and was maintained at $39.91 \mathrm{~g} / \mathrm{L}$ (Figure 4A). Ultimately, total glucose consumption was $56.60 \mathrm{~g}$, which was not significantly different from that in BEKW (Jang et al., 2012). Consistent with the similar glucose consumption in BEKW and mutant strains, cell growth was also unaffected by atp $G$ knockdown (Figure 4A; Jang et al., 2012). Production of the metabolites, acetate, butyrate, acetone, ethanol, and butanol, by BEKW-2866KD, was also analyzed and compared with that of BEKW (Jang et al., 2012). This analysis could be not confirmed significant changes in physiological characteristics (Figure 4). Furthermore, we found that the identified difference in residual butyrate between ATCC 824 and $824-2866 \mathrm{KD}$ was not repeated in the comparison between BEKW and BEKW-2866KD (Figure 4D).

The extracellular $\mathrm{pH}$ of BEKW and BEKW-2866KD, cultured while maintaining the $\mathrm{pH}$ above 5.0, was comparatively analyzed. During the solventogenic phase, $\mathrm{pH}$ steadily rose because of acid reassimilation in both BEKW and BEKW-2866KD fermentations. The extracellular $\mathrm{pH}$ in BEKW cultures reached 5.9, a value that was 

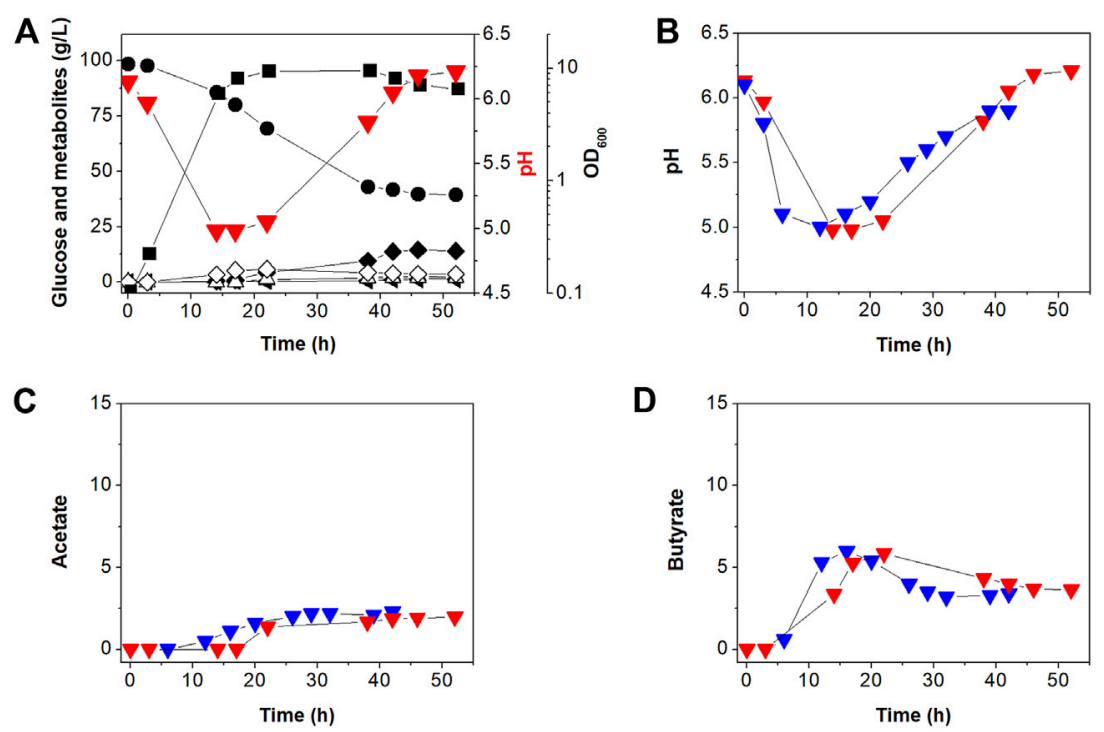

FIGURE 4 | Batch fermentation profiles of C. acetobutylicum BEKW-2866KD in bioreactor containing 2-L CGM (A). (A) Symbols are: $\bullet$, glucose; $\mathbf{\bullet}$, cell density $\left(\mathrm{OD}_{600}\right) ; \boldsymbol{\nabla}$, extracellular $\mathrm{pH} ; \triangle$, acetate; $\diamond$, butyrate; $\mathbf{\Lambda}$, acetone; $\mathbf{\Lambda}$, ethanol; and $\bullet$, butanol. Other reproduced bioreactor cultivation profiles are shown in Supplementary Figure S4. (B-D) Comparison of the external pH change (B), acetate (C), and butyrate (D) in batch fermentations between C. acetobutylicum BEKW (blue) and its mutant BEKW-2866KD (red). For this comparison, $\mathrm{pH}$, acetate, and butyrate values in the parent BEKW fermentation were obtained from our previous work (Jang et al., 2012).

maintained after $39 \mathrm{~h}$ (Jang et al., 2012). BEKW-2866KD reach a higher $\mathrm{pH}$ value of $\mathrm{pH} 6.54$, which was maintained after $46 \mathrm{~h}$ (Figure 4B). In other reproduced bioreactor cultivation, $\mathrm{pH} 6.89$ was observed at $46 \mathrm{~h}$ (Supplementary Figure S4). These results are similar to those obtained in comparisons between ATCC 824 and 824-2866KD, described above. The finding that atp $G$ knockdown caused no significant differences in cell growth, glucose consumption, or metabolites production indicates that ATPase is relatively minor than acid-forming pathway in ATP metabolism in C. acetobutylicum. However, the fact that ATPase knockdown similarly affected extracellular $\mathrm{pH}$ in atpG knockdown strains indicates that ATPase is affected to extracellular $\mathrm{pH}$ at the late stationary phase in C. acetobutylicum fermentation. Taken together, it seems that the external $\mathrm{pH}$ was affected by not only the residual acids but also other effectors, such as inhibition of proton pumping by ATPase. Depending on the situation, F-ATPase can reversibly synthesize or degrade ATP (Löbau et al., 1998; Bowler et al., 2006; Hayashi et al., 2012). ATP is hydrolyzed to create a proton gradient through the plasma membrane, while PMF is used for ATP synthesis (Costa et al., 2021). The increase in extracellular $\mathrm{pH}$ shown in this study is presumed to be due to inhibition of proton pumping across the membrane by knockdown of the atpG gene. This seems to be closely related to the recent report that ATPase is inhibited by butanol, which resulted in a low intracellular $\mathrm{pH}$ and reduction of PMF (Costa et al., 2021).

In this study, we first constructed the atp $G$ knockdown strains using the mobile group II intron system to investigate the role of the ATPase in C. acetobutylicum. Although other ATPasedisrupted non-clostridia organisms show prominent differences in ATP synthesis and cell growth, the atpG knockdown mutants of C. acetobutylicum ATCC 824 and
BEKW, 824-2866KD and BEKW-2866KD, respectively, showed no significant changes in physiological characteristics except extracellular $\mathrm{pH}$. The inference is that most ATP is produced through substrate-level phosphorylation in glycolysis and the acid-forming pathways in C. acetobutylicum. Detection of the ATP level may help to explain the phenomenon found in this work. As ATP and redox metabolism is complexly combined to biphasic fermentation in C. acetobutylicum, however, it is needed to approach it with a more elaborate strategy.

\section{DATA AVAILABILITY STATEMENT}

The original contributions presented in the study are included in the article/Supplementary Material, further inquiries can be directed to the corresponding authors.

\section{AUTHOR CONTRIBUTIONS}

SL and Y-SJ conceived the project. JI, HS, HL, and Y-SJ performed experiments. SK, Y-SL, HS, HL, YY, and Y-SJ were involved in analysis and interpretation of experimental data. SK, Y-SL, HS, HL, YY, SL and Y-SJ wrote the manuscript. All authors read and approved the final manuscript.

\section{FUNDING}

This work was supported by a grant from the Ministry of Science and ICT (MSIT) through the National Research Foundation 
(NRF) of Korea (NRF-2019R1A4A1029125). SL was supported by the Technology Development Program to Solve Climate Changes on Systems Metabolic Engineering for Biorefineries from the MSIT through the NRF of Korea (NRF2012M1A2A2026556 and NRF-2012M1A2A2026557).

\section{REFERENCES}

An, S. H., Choi, G.-S., and Ahn, J.-H. (2020). Biosynthesis of Fraxetin from Three Different Substrates Using Engineered Escherichia coli. Appl. Biol. Chem. 63, 55. doi:10.1186/s13765-020-00543-9

Baek, S.-A., Ahn, S. K., Kim, K. W., Choi, J., Kim, J., Ahn, J., et al. (2019). Metabolic Profiling Reveals Glucose and Fructose Accumulation in Gcr1 Knock-Out Mutant of Arabidopsis. Appl. Biol. Chem. 62, 23. doi:10.1186/s13765-0190427-3

Borghese, R., Turina, P., Lambertini, L., and Melandri, B. A. (1998). The atpIBEXF Operon Coding for the $\mathrm{F}_{0}$ Sector of the ATP Synthase from the Purple Nonsulfur Photosynthetic Bacterium Rhodobacter Capsulatus. Arch. Microbiol. 170, 385-388. doi:10.1007/s002030050657

Bowler, M. W., Montgomery, M. G., Leslie, A. G. W., and Walker, J. E. (2006). How Azide Inhibits ATP Hydrolysis by the F-ATPases. Proc. Natl. Acad. Sci. 103, 8646-8649. doi:10.1073/pnas.0602915103

Burger, M., Rein, S., Weber, S., Gräber, P., and Kacprzak, S. (2020). Distance Measurements in the $\mathrm{F}_{0} \mathrm{~F}_{1}$-ATP Synthase from E. coli Using smFRET and PELDOR Spectroscopy. Eur. Biophys. J. 49, 1-10. doi:10.1007/s00249-01901408-w

Causey, T. B., Zhou, S., Shanmugam, K. T., and Ingram, L. O. (2003). Engineering the Metabolism of Escherichia coli W3110 for the Conversion of Sugar to Redox-Neutral and Oxidized Products: Homoacetate Production. Proc. Natl. Acad. Sci. 100, 825-832. doi:10.1073/pnas.0337684100

Cho, C., Choe, D., Jang, Y.-S., Kim, K.-J., Kim, W. J., Cho, B.-K., et al. (2017). Genome Analysis of a Hyper Acetone-Butanol-Ethanol (ABE) Producing Clostridium acetobutylicum BKM19. Biotechnol. J. 12, 1600457. doi:10.1002/ biot. 201600457

Chun, J., and Sang, B.-I. (2020). Enzymatic Esterification under High-Pressure $\mathrm{CO} 2$ Conditions for In Situ Recovery of Butyric Acid from Anaerobic Fermenters. Biotechnol. Bioproc. E 25, 616-622. doi:10.1007/s12257-0200158-7

Cipriano, D. J., Wood, K. S., Bi, Y., and Dunn, S. D. (2006). Mutations in the Dimerization Domain of the B Subunit from the Escherichia coli ATP Synthase. J. Biol. Chem. 281, 12408-12413. doi:10.1074/jbc.m513368200

Costa, P., Usai, G., Re, A., Manfredi, M., Mannino, G., Bertea, C. M., et al. (2021). Clostridium Cellulovorans Proteomic Responses to Butanol Stress. Front. Microbiol. 12, 674639. doi:10.3389/fmicb.2021.674639

Dai, Z., Zhu, Y., Dong, H., Zhao, C., Zhang, Y., and Li, Y. (2020). Enforcing ATP Hydrolysis Enhanced Glycolysis and Promoted Solvent Production under Anaerobic Conditions. Microb. Cel Fact. 20, 149. doi:10.21203/rs.3.rs20422/v1

Du, G., Che, J., Wu, Y., Wang, Z., Jiang, Z., Ji, F., et al. (2021). Disruption of Hydrogenase Gene for Enhancing Butanol Selectivity and Production in Clostridium acetobutylicum. Biochem. Eng. J. 171, 108014. doi:10.1016/ j.bej.2021.108014

Externbrink, T., Hujer, S., Winzer, K., and Dürre, P. (2000). Sequence Analysis of the atp Operon of Clostridium acetobutylicum DSM 792 Encoding the $\mathrm{F}_{0} \mathrm{~F}_{1}$ ATP Synthase. DNA Seq. 11, 109-118. doi:10.3109/10425170009033977

Fast, A. G., and Papoutsakis, E. T. (2018). Functional Expression of the Clostridium Ljungdahlii Acetyl-Coenzyme A Synthase in Clostridium acetobutylicum as Demonstrated by a Novel In Vivo CO Exchange Activity En Route to Heterologous Installation of a Functional Wood-Ljungdahl Pathway. Appl. Environ. Microbiol. 84, e02307-02317. doi:10.1128/AEM.02307-17

Ferrándiz, M., and De La Campa, A. G. (2002). The Membrane-Associated $\mathrm{F}_{0} \mathrm{~F}_{1}$ ATPase Is Essential for the Viability of Streptococcus pneumoniae. FEMS Microbiol. Lett. 212, 133-138. doi:10.1016/s0378-1097(02)00717-6

Hayashi, S., Ueno, H., Shaikh, A. R., Umemura, M., Kamiya, M., Ito, Y., et al. (2012). Molecular Mechanism of ATP Hydrolysis in F1-ATPase Revealed by

\section{SUPPLEMENTARY MATERIAL}

The Supplementary Material for this article can be found online at: https://www.frontiersin.org/articles/10.3389/fbioe.2021.754250/ full\#supplementary-material

Molecular Simulations and Single-Molecule Observations. J. Am. Chem. Soc. 134, 8447-8454. doi:10.1021/ja211027m

Heap, J. T., Pennington, O. J., Cartman, S. T., Carter, G. P., and Minton, N. P. (2007). The ClosTron: a Universal Gene Knock-Out System for the Genus Clostridium. J. Microbiol. Methods 70, 452-464. doi:10.1016/ j.mimet.2007.05.021

Im, H. S., Kim, C., Song, Y. E., Baek, J., Im, C. H., and Kim, J. R. (2019). Isolation of Novel CO Converting Microorganism Using Zero Valent Iron for a Bioelectrochemical System (BES). Biotechnol. Bioproc. E 24, 232-239. doi:10.1007/s12257-018-0373-7

Im, H., An, T., Kwon, R., Park, S., and Kim, Y.-K. (2021). Effect of Organic Nitrogen Supplements on Syngas Fermentation Using Clostridium Autoethanogenum. Biotechnol. Bioproc. Eng. 26, 476-482. doi:10.1007/ s12257-020-0221-4

Iwamoto, A., Miki, J., Maeda, M., and Futai, M. (1990). H(+)-ATPase Gamma Subunit of Escherichia coli. Role of the Conserved Carboxyl-Terminal Region. J. Biol. Chem. 265, 5043-5048. doi:10.1016/s0021-9258(19)34081-5

Jang, Y. S., Lee, J. Y., Lee, J., Park, J. H., Im, J. A., Eom, M. H., et al. (2012). Enhanced Butanol Production Obtained by Reinforcing the Direct Butanol-Forming Route in Clostridium acetobutylicum. mBio 3, e00314-00312. doi:10.1128/ mBio.00314-12

Jang, Y.-S., Han, M.-J., Lee, J., Im, J. A., Lee, Y. H., Papoutsakis, E. T., et al. (2014a). Proteomic Analyses of the Phase Transition from Acidogenesis to Solventogenesis Using Solventogenic and Non-solventogenic Clostridium acetobutylicum Strains. Appl. Microbiol. Biotechnol. 98, 5105-5115. doi:10.1007/s00253-014-5738-z

Jang, Y.-S., Im, J. A., Choi, S. Y., Lee, J. I., and Lee, S. Y. (2014b). Metabolic Engineering of Clostridium acetobutylicum for Butyric Acid Production with High Butyric Acid Selectivity. Metab. Eng. 23, 165-174. doi:10.1016/ j.ymben.2014.03.004

Jensen, P. R., and Michelsen, O. (1992). Carbon and Energy Metabolism of atp Mutants of Escherichia coli. J. Bacteriol. 174, 7635-7641. doi:10.1128/ jb.174.23.7635-7641.1992

Jiang, Y., Wu, R., Lu, J., Dong, W., Zhou, J., Zhang, W., et al. (2021). Quantitative Proteomic Analysis to Reveal Expression Differences for Butanol Production from Glycerol and Glucose by Clostridium Sp. Strain CT7. Microb. Cell Fact. 20, 12. doi:10.1186/s12934-021-01508-3

Kang, C., Sun, F., Yan, L., Li, R., Bai, J., and Caetano-Anollés, G. (2019). GenomeWide Identification and Characterization of the Vacuolar $\mathrm{H}^{+}$-ATPase Subunit H Gene Family in Crop Plants. Ijms 20, 5125. doi:10.3390/ijms20205125

Kim, S., Jang, Y.-S., Ha, S.-C., Ahn, J.-W., Kim, E.-J., Hong Lim, J., et al. (2015). Redox-switch Regulatory Mechanism of Thiolase from Clostridium acetobutylicum. Nat. Commun. 6, 8410. doi:10.1038/ncomms9410

Kim, J.-T., Yi, G., Kim, M.-J., Son, B.-Y., Bae, H.-H., Go, Y. S., et al. (2020). Glycolysis Stimulation and Storage Protein Accumulation Are Hallmarks of maize (Zea mays L.) Grain Filling. Appl. Biol. Chem. 63, 54. doi:10.1186/s13765020-00538-6

Koch-Koerfges, A., Kabus, A., Ochrombel, I., Marin, K., and Bott, M. (2012). Physiology and Global Gene Expression of a Corynebacterium glutamicum $\triangle \mathrm{F}_{1} \mathrm{~F}_{\mathrm{O}}$-ATP Synthase Mutant Devoid of Oxidative Phosphorylation. Biochim. Biophys. Acta (BBA) - Bioenerg. 1817, 370-380. doi:10.1016/ j.bbabio.2011.10.006

Koebmann, B. J., Nilsson, D., Kuipers, O. P., and Jensen, P. R. (2000). The Membrane-Bound $\mathrm{H}^{+}$-ATPase Complex Is Essential for Growth of Lactococcus lactis. J. Bacteriol. 182, 4738-4743. doi:10.1128/jb.182.17.47384743.2000

Koebmann, B. J., Solem, C., Pedersen, M. B., Nilsson, D., and Jensen, P. R. (2002a). Expression of Genes Encoding $F_{1}$-ATPase Results in Uncoupling of Glycolysis from Biomass Production in Lactococcus lactis. Appl. Environ. Microbiol. 68, 4274-4282. doi:10.1128/aem.68.9.4274-4282.2002 
Koebmann, B. J., Westerhoff, H. V., Snoep, J. L., Nilsson, D., and Jensen, P. R. (2002b). The Glycolytic Flux in Escherichia coli Is Controlled by the Demand for ATP. J. Bacteriol. 184, 3909-3916. doi:10.1128/jb.184.14.3909-3916.2002

Kwon, S. W., Paari, K. A., Malaviya, A., and Jang, Y.-S. (2020). Synthetic Biology Tools for Genome and Transcriptome Engineering of Solventogenic Clostridium. Front. Bioeng. Biotechnol. 8, 282. doi:10.3389/fbioe.2020.00282

Lai-Zhang, J., Xiao, Y., and Mueller, D. M. (1999). Epistatic Interactions of Deletion Mutants in the Genes Encoding the $\mathrm{F}_{1}$-ATPase in Yeast Saccharomyces cerevisiae. EMBO J. 18, 58-64. doi:10.1093/emboj/18.1.58

Lee, J. S., Paje, L. A., Choi, W.-H., Cho, E. J., Kim, H. Y., Jacinto, S. D., et al. (2020). Validation of an Optimized HPLC/UV Method for the Quantification of Flavonoids in Lotus. Appl. Biol. Chem. 63, 84. doi:10.1186/s13765-02000568-0

Li, S., Huang, L., Ke, C., Pang, Z., and Liu, L. (2020). Pathway Dissection, Regulation, Engineering and Application: Lessons Learned from Biobutanol Production by Solventogenic Clostridia. Biotechnol. Biofuels 13, 39. doi:10.1186/s13068-020-01674-3

Löbau, S., Weber, J., and Senior, A. E. (1998). Catalytic Site Nucleotide Binding and Hydrolysis in $\mathrm{F}_{1}$ Fo-ATP Synthase. Biochemistry 37, 10846-10853. doi:10.1021/ bi9807153

Lone, S. R., Kumar, V., Seay, J. R., Englert, D. L., and Hwang, H. T. (2020). Mass Transfer and Rheological Characteristics in a Stirred Tank Bioreactor for Cultivation of Escherichia coli BL21. Biotechnol. Bioproc. Eng. 25, 766-776. doi:10.1007/s12257-020-0028-3

Lütke-Eversloh, T. (2014). Application of New Metabolic Engineering Tools for Clostridium acetobutylicum. Appl. Microbiol. Biotechnol. 98, 5823-5837. doi:10.1007/s00253-014-5785-5

Mermelstein, L. D., and Papoutsakis, E. T. (1993). In Vivo Methylation in Escherichia coli by the Bacillus Subtilis Phage Phi 3T I Methyltransferase to Protect Plasmids from Restriction upon Transformation of Clostridium acetobutylicum ATCC 824. Appl. Environ. Microbiol. 59, 1077-1081. doi:10.1128/aem.59.4.1077-1081.1993

Mukherjee, S., and Warshel, A. (2015). Dissecting the Role of the $\gamma$-subunit in the Rotary-Chemical Coupling and Torque Generation of $\mathrm{F}_{1}$-ATPase. Proc. Natl. Acad. Sci. U.S.A 112, 2746-2751. doi:10.1073/pnas.1500979112

Nölling, J., Breton, G., Omelchenko, M. V., Makarova, K. S., Zeng, Q., Gibson, R., et al. (2001). Genome Sequence and Comparative Analysis of the SolventProducing Bacterium Clostridium acetobutylicum. J. Bacteriol. 183, 4823-4838. doi:10.1128/JB.183.16.4823-4838.2001

Poehlein, A., Solano, J. D. M., Flitsch, S. K., Krabben, P., Winzer, K., Reid, S. J., et al. (2017). Microbial Solvent Formation Revisited by Comparative Genome Analysis. Biotechnol. Biofuels 10, 58. doi:10.1186/s13068-017-0742-z

Santana, M., Ionescu, M. S., Vertes, A., Longin, R., Kunst, F., Danchin, A., et al. (1994). Bacillus subtilis $\mathrm{F}_{0} \mathrm{~F}_{1}$ ATPase: DNA Sequence of the atp Operon and Characterization of atp Mutants. J. Bacteriol. 176, 6802-6811. doi:10.1128/ jb.176.22.6802-6811.1994

Sekine, H., Shimada, T., Hayashi, C., Ishiguro, A., Tomita, F., and Yokota, A. (2001). $\mathrm{H}^{+}$-ATPase Defect in Corynebacterium glutamicum Abolishes Glutamic Acid Production with Enhancement of Glucose Consumption Rate. Appl. Microbiol. Biotechnol. 57, 534-540. doi:10.1007/s002530100778
Shah, N. B., and Duncan, T. M. (2015). Aerobic Growth of Escherichia coli Is Reduced, and ATP Synthesis Is Selectively Inhibited when Five C-terminal Residues Are Deleted from the $\epsilon$ Subunit of ATP Synthase. J. Biol. Chem. 290, 21032-21041. doi:10.1074/jbc.m115.665059

Shao, L., Hu, S., Yang, Y., Gu, Y., Chen, J., Yang, Y., et al. (2007). Targeted Gene Disruption by Use of A Group II Intron (Targetron) Vector in Clostridium acetobutylicum. Cell Res. 17, 963-965. doi:10.1038/cr.2007.91

Shin, K., Nakamoto, R. K., Maeda, M., and Futai, M. (1992). $\mathrm{F}_{0} \mathrm{~F}_{1}$-ATPase Gamma Subunit Mutations Perturb the Coupling between Catalysis and Transport. J. Biol. Chem. 267, 20835-20839. doi:10.1016/s0021-9258(19)36763-8

Shin, Y.-A., Choi, S., and Han, M. (2021). Simultaneous Fermentation of Mixed Sugar by a Newly Isolated Clostridium beijerinckii GSC1. Biotechnol. Bioproc. Eng. 26, 137-144. doi:10.1007/s12257-020-0183-6

Thi, H. N., Park, S., Li, H., and Kim, Y.-K. (2020). Medium Compositions for the Improvement of Productivity in Syngas Fermentation with Clostridium autoethanogenum. Biotechnol. Bioproc. E. 25, 493-501. doi:10.1007/s12257019-0428-4

Tremblay, P. L., Zhang, T., Dar, S. A., Leang, C., and Lovley, D. R. (2012). The Rnf Complex of Clostridium ljungdahlii Is a Proton-Translocating ferredoxin: $\mathrm{NAD}^{+}$Oxidoreductase Essential for Autotrophic Growth. mBio 4, e00406-12. doi:10.1128/mBio.00406-12

Weber, E. R., Rooks, R. S., Shafer, K. S., Chase, J. W., and Thorsness, P. E. (1995). Mutations in the Mitochondrial ATP Synthase Gamma Subunit Suppress A Slow-Growth Phenotype of yme1 Yeast Lacking Mitochondrial DNA. Genetics 140, 435-442. doi:10.1093/genetics/140.2.435

Zhang, X. C., and Zhang, H. (2019). P-type ATPases Use a Domain-Association Mechanism to Couple ATP Hydrolysis to Conformational Change. Biophys. Rep. 5, 167-175. doi:10.1007/s41048-019-0087-1

Zharova, T. V., and Vinogradov, A. D. (2017). Functional Heterogeneity of Fo $\cdot \mathrm{F}_{1} \mathrm{H}^{+}$-ATPase/synthase in Coupled Paracoccus denitrificans Plasma Membranes. Biochim. Biophys. Acta (BBA) - Bioenerg. 1858, 939-944. doi:10.1016/j.bbabio.2017.08.006

Conflict of Interest: The authors declare that the research was conducted in the absence of any commercial or financial relationships that could be construed as a potential conflict of interest.

Publisher's Note: All claims expressed in this article are solely those of the authors and do not necessarily represent those of their affiliated organizations, or those of the publisher, the editors and the reviewers. Any product that may be evaluated in this article, or claim that may be made by its manufacturer, is not guaranteed or endorsed by the publisher.

Copyright (c) 2021 Jang, Seong, Kwon, Lee, Im, Lee, Yoon and Lee. This is an openaccess article distributed under the terms of the Creative Commons Attribution License (CC BY). The use, distribution or reproduction in other forums is permitted, provided the original author(s) and the copyright owner(s) are credited and that the original publication in this journal is cited, in accordance with accepted academic practice. No use, distribution or reproduction is permitted which does not comply with these terms. 